\title{
Mikrowellenablation und Immunaktivierung in der Behandlung rezidivierender Lungenmetastasen eines Kolorektalkarzinoms: Ein Fallbericht
}

\author{
Magnus Bäcklund ${ }^{\mathrm{a}}$ Jacob Freedman ${ }^{\mathrm{b}}$ \\ a Department of Medicine Solna, Karolinska University Hospital, CMM, Karolinska Institutet, Stockholm, Sweden; \\ ${ }^{b}$ Division of Surgery and Urology, Department of Clinical Sciences at Danderyd University Hospital, Karolinska Institutet, Stockholm, Sweden
}

\author{
Schlüsselwörter \\ Kolorektalkarzinom · Lunge · Metastasen · Mikrowellen · \\ Navigierte Ablation · Cascination · Resektion · Bestrahlung · \\ Immunaktivierung $\cdot$ Pembrolizumab
}

\begin{abstract}
Zusammenfassung
Wir berichten über eine Patientin mit Metastasen eines Kolorektalkarzinoms, die auf die Lunge begrenzt waren und mehrfach reseziert wurden, bis die Resektion keine therapeutische Option mehr darstellte; daraufhin wurde eine stereotaktische Körperbestrahlung durchgeführt bis auch diese Option ausgeschöpft war. Anschließend erfolgte eine erfolgreiche Behandlung mit multiplen
\end{abstract}

Mikrowellenablationen in Kombination mit einer immunaktivierenden Therapie, die am programmed cell death 1 receptor (PD-1) angreift und möglicherweise einen starken abscopalen Effekt auslöst. Im Folgenden werden Verfahren, Dosierungen und radiologische Befunde dargestellt.

(c) 2017 S. Karger GmbH, Freiburg

\section{Einleitung}

Das Kolorektalkarzinom ist die dritthäufigste Krebsart weltweit und außerdem die vierthäufigste krebsbedingte Todesursache [1]. Bei der Erstvorstellung des Patienten kommt dem Staging entscheidende Bedeutung zu, da es prognostisch wichtige Informationen liefert und als Orientierungshilfe für die Therapieentscheidungen dient. Eine kurative Behandlung ist indiziert, wenn die vollständige Entfernung möglich scheint. Die Krankheit ist bei der Hälfte der Patienten zum Zeitpunkt der Diagnose bereits ausgebreitet oder streut im weiteren Verlauf Metastasen. Etwa $30 \%$ entwickeln Lebermetastasen [2], und bei ungefähr $20 \%$ kommt es zu Lungenmetastasen [3, 4], die beiden häufigsten Lokalisationen für Fernmetastasen. Das Auftreten von Metastasen in der Lunge ist mit einer besseren Prognose verbunden als in anderen Körperregionen, und die Krankheitsprogression verläuft langsamer [5]. Therapie der ersten Wahl ist die Resektion mit einer möglichen adjuvanten stereotaktischen Körperbestrahlung (stereotactic body radiation therapy, SBRT) oder thermoablativen Prozeduren als Salvage-Verfahren. Die Leitlinien empfehlen häufig eine Chemotherapie mit Remission oder stabiler Erkrankung bevor ein Therapieversuch mit einer potenziell kurativen Behandlung der Metastasen unternommen wird [6]. Ein vollkommen neues Forschungsfeld hat sich mit neuen immunmodulierenden Medikamenten aufgetan, die allerdings angreifbare antigene Eigenschaften auf der Tumoroberfläche benötigen, um wirken zu können. Dies ist bei Kolorektalkarzinomen mit Mikrosatelliten-Instabilität in ihrem Genom zu beobachten. Die Mikrosatelliten-Instabilität führt zu einer veränderten Expression der Oberflächenantigene und ermöglicht dadurch eine zytotoxische T-Zellaktivität, welche durch Medikamente mit Wirkung am programmed cell death 1 receptor (PD-1) «entsperrt»

\section{KARGER}

Fax +4976145207 14

information@karger.com

www.karger.com 
Abb. 1. a Ein 3D-Rekonstruktion des knöchernen Thorax und der 6 Metastasen mit den geplanten Trajektorien für die Ablationselektroden. Ansicht von unten, Trajektorien vom dorsalen Eintrittspunkt. b Stereotaktische Platzierung der Mikrowellenelektrode im zu abladierenden Tumor.
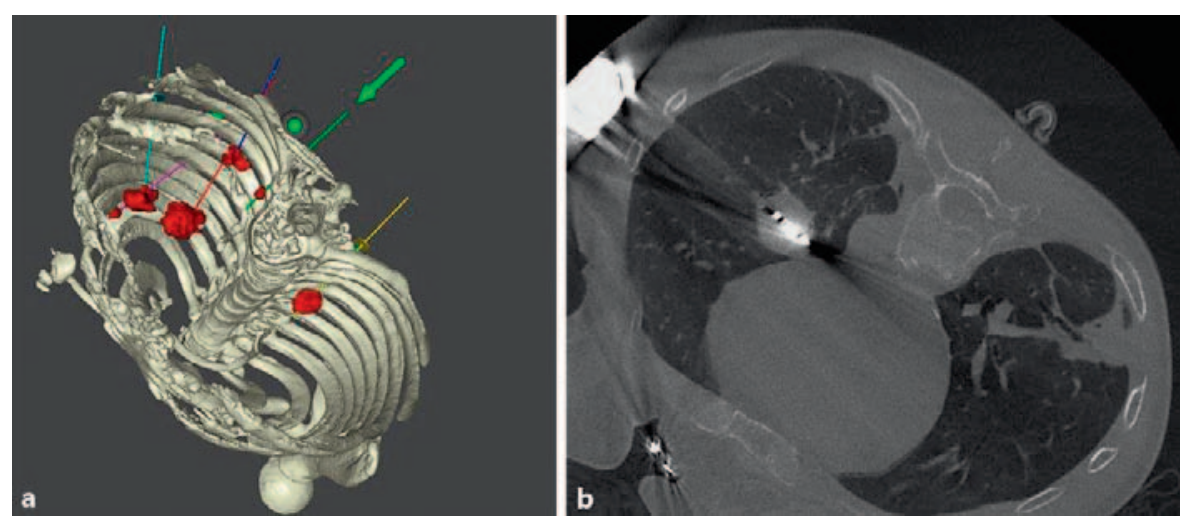

werden kann [6, 7]. Eine andere Methode, neue Antigene auf Tumoren $\mathrm{zu}$ «öffnen» ist die lokale Zerstörung mithilfe ablativer Verfahren wie Kryotherapie, Radiofrequenz (RF)-Ablation oder Mikrowellenablation. Mikrowellen haben den Vorteil, dass sie die Energie schnell liefern, wodurch die Ablationsdauer kurz gehalten werden kann und der kühlende Blutfluss durch benachbarte Gefäße nur einen minimalen Einfluss hat $[8,9]$. Zudem ist bei der Behandlung der Lunge, anders als bei der RF-Ablation, keine Gewebeleitfähigkeit erforderlich, da sich Mikrowellen über die Luft in den Alveolen verbreiten können. Wenn eine gute Immunreaktion induziert werden kann, ist bisweilen ein allgemeiner Effekt auf das gesamte Tumorgewebe, ein sogenannter abscopaler Effekt, zu beobachten, der bei Tieren nachgewiesen wurde, bei denen eine Immunmodulation mittels lokal ablativer Verfahren durchgeführt wurde [10].

\section{Fallbericht}

Wir berichten über den Fall einer 51-jährigen Frau, die in den 1980er Jahren als Teenager ein Guillain-Barré-Syndrom, vermutlich als Folge einer Neuroborreliose, hatte und in den 1990er Jahren ein malignes Melanoma in situ. Sie hat eine schriftliche Einwilligungserklärung für die Veröffentlichung dieses Berichts erteilt. Die Patientin wurde wegen Sphincterverletzungen durch die Geburt wiederholt rektal operiert, und 2011 wurde ein blutendes Rektumkarzinom im Stadium T4 festgestellt. Fernmetastasen fanden sich nicht, und im Rahmen einer multidisziplinären Tumorkonferenz wurde entschieden, die Patientin mit kurativer Zielsetzung zu behandeln. Sie erhielt eine neoadjuvante Chemotherapie mit Fluorouracil und Leucovorin gefolgt von einer Bestrahlung des Rektums mit 50,4 Gy. Ein halbes Jahr nach der Diagnose erfolgte die Resektion des Tumors. Der postoperative Verlauf war bis auf eine oberflächliche Wundinfektion unauffällig und nach dreiwöchiger stationärer Behandlung konnte die Patientin entlassen werden. Zwei Wochen später wurde sie erneut stationär aufgenommen wegen einer Tako-Tsubo-Kardiomyopathie und intestinalen Obstruktion, die eine erneute Operation mit Resektion eines kurzen Dünndarmsegments erforderte. Nach einem Jahr wurden die ersten Lungenmetastasen festgestellt, mit einer $2 \mathrm{~cm}$ großen Metastase im rechten Unterlappen und einem $5 \mathrm{~mm}$ großen Tumor im linken Unterlappen. Nach drei pulmonalen Resektions- operationen traten während der Nachsorge weitere Lungenmetastasen auf. Die Patientin erhielt eine Zweitlinienchemotherapie mit Oxaliplatin, die eine Verkleinerung der Läsionen bewirkte. Anschließend, drei Jahre nach Resektion des Primärtumors, erfolgte eine lokale Behandlung mit SBRT $3 \times 17$ Gy wegen zwei rechtsseitiger Metastasen und eine Resektion des linken Lungenflügels. Drei Monate später wurde eine neue Lungenmetastase diagnostiziert und mit SBRT $8 \times 7$ Gy behandelt. Sechs Monate später traten zwei weitere Lungenläsionen auf und eine Drittlinienchemotherapie mit Irinotecan wurde eingeleitet, jedoch wegen nachgewiesener Tumorprogression nach drei Monaten abgesetzt. Man entschied, eine PD-1-Therapie zu beginnen, ohne das endgültige Untersuchungsergebnis hinsichtlich der Mikrosatelliteninstabilität des Tumors abzuwarten (die Behandlung erfolgte nicht im Rahmen einer klinischen Studie, da die einzige verfügbare Studie in Schweden noch nicht geöffnet war). Weitere Resektionen waren nicht möglich und die verabreichte Strahlendosis schloss eine erneute SBRT aus. Nach den ersten drei Dosen von Pembrolizumab (in der Standarddosierung von $2 \mathrm{mg} / \mathrm{kg}$ alle drei Wochen) fanden sich insgesamt sechs bestätigte Metastasen auf beiden Seiten. In den CT-Aufnahmen zeigte sich eine Größenzunahme aller Lungenmetastasen, jedoch ohne neue Läsionen. Als einzige Nebenwirkung trat bei der Patientin Fatigue auf. Die frühe Beurteilung sprach nicht gegen eine Fortsetzung der immunologischen Therapie, doch wurde vorgeschlagen, zunächst eine lokale ablative Behandlung durchzuführen, da diese bei Lebertumoren mittlerweile zum Standard gehört und ihre Wirksamkeit in der Behandlung von Lungenläsionen durch eine zunehmende Anzahl internationaler Veröffentlichungen gestützt wird [9, 11-13]. Die Ablation der als behandelbar eingestuften Metastasen erfolgte nach zwei weiteren Dosen Pembrolizumab in zwei Sitzungen im Abstand von drei Wochen mithilfe eines computergestützen Systems (CAS-one; Cascination AG, Bern, Schweiz) und Mikrowellen (Angiodynamics) unter Immobilisierung der Lunge mit Hochfrequenzbeatmung (high-frequency jet ventilation), wie in einer früheren Veröffentlichung berichtet [14]. Nach den Ablationen blieb die Patientin über Nacht im Krankenhaus. Bei der ersten Ablationssitzung kam es zu einem Pneumothorax, der mittels Thoraxdrainage (16F) behandelt wurde, die nach Abklemmen am nächsten Tag entfernt werden konnte. Aufnahmen der Intervention sind in Abbildung 1 dargestellt. 
Abb. 2. a Ein Post-Ablationsbild mit multiplen kleinen Arealen fokaler Reaktionen, sogenannte Pseudotumoren, als Reaktion auf die Immuntherapie. b Das gleiche Areal drei Monate später nach Behandlung mit Kortikosteroiden.
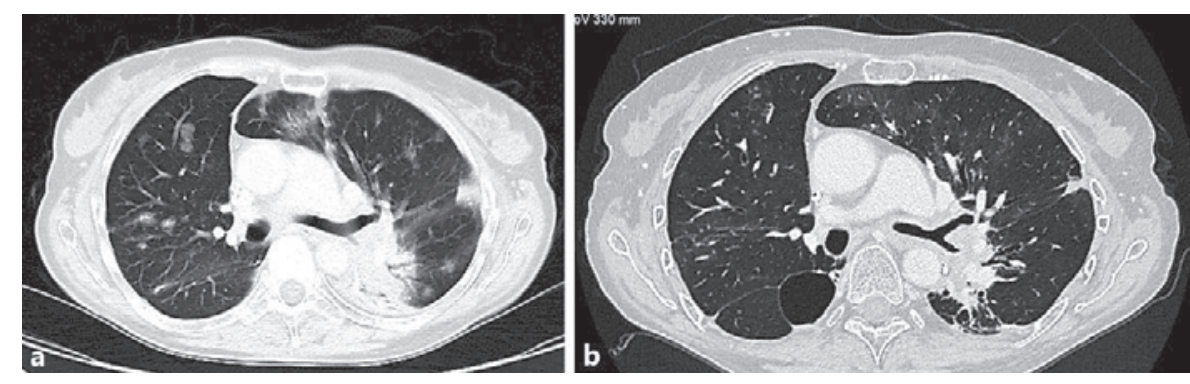

Nach zwei Wochen wurde die PD-1-Behandlung wieder aufgenommen. Nach dem sechsten Pembrolizumab-Zyklus klagte die Patientin über Schmerzen im Bereich der Leber sowie Appetitminderung und einen leichten Gewichtsverlust. Weitere Untersuchungen ergaben keine Zeichen von Lebermetastasen oder einer Leberschädigung, allerdings einen Abfall des Serumalbuminspiegels sowie erhöhte ESR- und CRP-Werte. Dieser Anstieg der Entzündungsparameter wurde als mögliche Reaktion auf die PD1-Hemmung gewertet und mit Betamethason behandelt. Für eine bessere Schmerzkontrolle wurde das analgetische Standardregime um Amitriptylin erweitert, da man eine neurogene Schmerzkomponente vermutete. Nach drei Wochen konnte die siebte Dosis Pembrolizumab verabreicht werden. Zwei Wochen später berichtete die Patientin über eine Schwäche im linken Unterschenkel, Tremor der Finger und Taubheitsgefühl auf der ulnaren Seite der rechten Hand. Der hinzugezogene Neurologe schloss den Verdacht auf neue Metastasen oder eine neurologische Systemerkrankung aus. Die Symptome wurden als Nebenwirkungen von Pembrolizumab eingestuft, wobei die zugrunde liegenden Mechanismen nicht vollständig klar sind. Die KortikosteroidDosis wurde erhöht, und die Verabreichung des achten Pembrolizumab-Zyklus verlief ohne Verschlimmerung der Symptome. Abbildung 2 zeigt Aufnahmen einer Kontroll-CT, auf denen eine initiale Entzündungsreaktion mit fokalen Läsionen, sogenannten Pseudotumoren, zu sehen ist, die im weiteren Verlauf verschwanden. Nach achtmonatigem Follow-up waren keine Anzeichen für neue oder rezidivierte Lungenmetastasen nachweisbar.

Derzeit bestehen bei der Patientin noch neurologische Symptome, die trotz Verabreichung höherer Steroiddosierungen nicht zurückgehen. Die andere Komplikation ist das Auftreten diffuser nicht-maligner Läsionen in beiden Lungenflügeln, die als entzündlich oder infektiös bedingt gewertet werden. Diese Beobach- tungen waren begleitet von Kurzatmigkeit und Problemen beim Gehen längerer Strecken. Die Steroidbehandlung brachte ebenso nur eine teilweise Linderung der Symptome, wie die Gabe von Antibiotika, die sowohl grampositive Bakterien als auch Pneumocystis carinii abdeckten. Weitere Untersuchungen laufen derzeit. Wie bereits erwähnt, sind die Lungenmetastasen nicht rezidiviert, sodass die Patientin gegenwärtig mit der antitumoralen Therapie pausiert.

\section{Schlussfolgerung}

Der vorgestellte Fall verdeutlicht, welche Möglichkeiten die Anwendung stereotaktisch navigierter Ablationselektroden zur Mikrowellenablation multipler Lungenmetastasen bietet. Darüber hinaus liefert er Hinweise auf mögliche immunologische Benefits, wenn die ablative Therapie mit einer immunaktivierenden Therapie mittels PD-1-hemmenden Medikamenten wie Pembrolizumab kombiniert wird. Hierzu wurden kürzlich vielversprechende frühe klinische Ergebnisse einer chinesischen Studie veröffentlicht [15]. Diese Kombination könnte eine ganze Bandbreite neuer Möglichkeiten auf dem Gebiet der Onkologie eröffnen, wobei jedoch alle Anstrengungen unternommen werden sollten, eine prospektive kontrollierte Vorgehensweise zu gewährleisten.

\section{Erklärung zu ethischen Konflikten}

Die schriftliche Einwilligungserklärung der Patientin liegt vor.

\section{Disclosure Statement}

Die Autoren erklären, dass keine Interessenkonflikte bestehen.

\section{Literatur}

1 Ferlay J, Soerjomataram I, Dikshit R, Eser S, Mathers C, Rebelo M, et al: Cancer incidence and mortality worldwide: sources, methods and major patterns in GLOBOCAN 2012. Int J Cancer 2015;136:E359-E386.

2 Hackl C, Neumann P, Gerken M, Loss M, Klinkhammer-Schalke M, Schlitt HJ: Treatment of colorectal liver metastases in Germany: a ten-year population-based analysis of 5,772 cases of primary colorectal adenocarcinoma. BMC Cancer 2014; 14:810.
3 Mitry E, Guiu B, Cosconea S, Jooste V, Faivre J, Bouvier A-M: Epidemiology, management and prognosis of colorectal cancer with lung metastases: a 30-year population-based study. Gut 2010;59:1383-1388.

4 Neo EL, Beeke C, Price T, Maddern G, Karapetis C, Luke C, et al: South Australian clinical registry for metastatic colorectal cancer. ANZ J Surg 2011;81:352-357.

5 Kim HK: Pulmonary metastasectomy for colorectal cancer: how many nodules, how many times? World J Gastroenterol 2014;20:6133.
6 ESMO Guidelines Working Group; Van Cutsem EJD: Advanced colorectal cancer: ESMO clinical recommendations for diagnosis, treatment and follow-up. Ann Oncol 2007;18(suppl 2):ii25-ii26.

7 Link JT, Overman MJ: Immunotherapy progress in mismatch repair-deficient colorectal cancer and future therapeutic challenges. Cancer J Sci Am 2016;22:190-195. 
8 Vogl TJ, Naguib NNN, Lehnert T, Nour-Eldin N-EA: Radiofrequency, microwave and laser ablation of pulmonary neoplasms: clinical studies and technical considerations - review article. Eur J Radiol 2011;77:346-357.

9 Vogl TJ, Eckert R, Naguib NNN, Beeres M, Gruber-Rouh T, Nour-Eldin N-EA: Thermal ablation of colorectal lung metastases: retrospective comparison among laser-induced thermotherapy, radiofrequency ablation, and microwave ablation. AJR Am J Roentgenol 2016;207:1340-1349.

10 den Brok MHMGM, Sutmuller RPM, van der Voort R, Bennink EJ, Figdor CG, Ruers TJM, et al: In situ tumor ablation creates an antigen source for the generation of antitumor immunity. Cancer Res 2004;64:4024-4029.

11 Ko W-C, Lee Y-F, Chen Y-C, Chien N, Huang Y-S, Tseng Y-H, et al: CTguided percutaneous microwave ablation of pulmonary malignant tumors. J Thorac Dis 2016;8:S659-S665.

12 Healey TT, March BT, Baird G, Dupuy DE: Microwave ablation for lung neoplasms: a retrospective analysis of long-term results. J Vasc Interv Radiol 2017;28:206-211.

13 Ierardi AM, Coppola A, Lucchina N, Carrafiello G: Treatment of lung tumours with high-energy microwave ablation: a single-centre experience. Med Oncol 2017;34:5.

14 Freedman J, Harbut P: Navigated percutaneous lung ablation under highfrequency jet ventilation of a metastasis from a Wilms' tumour: a paediatric case report. Case Rep Oncol 2016;9:400-404.

15 Shi L, Chen L, Wu C, Zhu Y, Xu B, Zheng X, et al: PD-1 blockade boosts radiofrequency ablation-elicited adaptive immune responses against tumor. Clin Cancer Res 2016; 22:1173-1184. flutiform 50 Mikrogramm/5 Mikrogramm, 125 Mikrogramm/5 Mikrogramm, 250 Mikrogramm/10 Mikrogramm pro Sprühstoß krogramm, 250 Mikrogramm/10 Mikrogramm pro Spruhsto Druckgasinhalation, Suspension. Wirkstofte. Futicason-17-propionat, Formoterol umarat-Dhydrat (Ph.Eur.). Verschreibungspplichtig. Zusammensetzung: Arzhellich wiksarne Bestandele. Jeder Sprahsto flutiform 50 Mikrogramm/5 Mikrogramm (abgegeben aus dem Ventil enthält: $50 \mu \mathrm{g}$ Fluticason-17-propionat und $5 \mu \mathrm{g}$ Formoterolfumarat Dihydrat (Ph.Eur.). Dies entspricht einer zugeführten Dosis (aus dem Mundstuck) von etwa $46 \mu \mathrm{gg}$ Fluticason-17-propionat $/ 4,5 \mu \mathrm{g}$ Formoterolfumarat-Dihydrat (Ph.Eur.). Jeder Sprühstoß flutiform 125 Mikrogramm/5 Mikrogramm (abgegeben aus dem Ventil) enthält: 12.5 $\mu \mathrm{g}$ Fluticason-17-propionat und $5 \mu \mathrm{g}$ Formoterolfumarat-Dihydrat (Ph Eur.). Dies entspricht einer zugeführten Dosis (aus dem Mundstück von etwa $115 \mu \mathrm{g}$ Fluticason-17-propionat/4,5 $\mu \mathrm{g}$ FormoterolfumaratDihydrat (Ph.Eur.). Jeder Sprühstoß flutiform 250 Mikrogramm/10 Mikrogramm (abgegeben aus dem Ventil) enthält: $250 \mu \mathrm{g}$ Fluticason17 -propionat und $10 \mu \mathrm{g}$ Formoterolfumarat-Dihydrat (Ph.Eur.). Dies entspricht einer zugeführten Dosis (aus dem Mundstück) von etwa $230 \mu \mathrm{g}$ Fluticason-17-propionat/9 $\mu \mathrm{g}$ Formoterolfumarat-Dihydrat (Ph.Eur.). Sonstige Bestandteile: Apafluran, Natriumcromoglicat (Ph. Eur.), Ethanol. Anwendungsgebiete: Die Fixkombination aus Fluticason-17-propionat und Formoterolfumarat-Dihydrat (flutiform) wird angewendet zur regelmäßigen Behandlung vo bronchiale in Fällen, in denen ein Kombinationspräparat (ein inhalatives Kortikosteroid und ein langwirksamer Beta-2-Agonist) angezeigt ist: Bei Patienten, die mit inhalativen Kortikosteroiden und bedarfsweise angewendeten, kurzwirksamen inhalativen Beta2-Agonisten nicht ausreichend eingestellt sind. Oder bei Patienten die bereits mit einem inhalativen Kortikosteroid und einem langwirksamen Beta-2-Agonisten adäquat eingestellt sind flutiform 50 Mikrogramm/5 Mikrogramm und flutiform 125 Mikrogramm/5 Mikrogramm pro Sprühstoß werden angewendet bei Erwachsenen und Jugendlichen ab 12 Jahren. flutiform 250 Mikrogramm/10 werden Gegenanzeigen: Überemnfindlichkeit gegen die Wirkstoffe oder einen der sonstigen Bestandteile Nebenwirkungen Infektionen und parasitäre Erkrankungen: Selten : Ora Kandidose, orale Pilzinfektionen, Sinusitis. Stoffwechsel- und Ernährungsstörungen: Selten: Hyperglykämie. Psychiatrische Erkrankungen: Gelegentlich. Schlafstörungen ind. Schlaflosigkeit. Erkrankungen: Gelegentlich: Schlaststorungen incl. Schlaflosigkeit. Selten: Ungewöhnliche Träume, Agitation. Nicht bekannt: Psychomotorische Hyperaktivitat, Angstgefuhle, Depression, Reizbarkelt und Verhaltensänderungen, (überwiegend bei Kindern). Erkrankun gen des Nervensystems: Gelegentlich: Koptschmerzen, Tremor, Benommenheit. Selten: Dysgeusie. Erkrankungen des Ohrs und des Labyrinths: Selten: Vertigo. Herzerkran Selten: Angina pectoris, Tachykardie. Gefäßerkrankungen: Selten: Hypertonie. Erkrankungen der Atemwege, des Brustraums und Mediastinums: Gelegentlich: Asthmaexazerbation, Dysphonie, Rachenreizung. Selten: Dyspnoe, Husten. Erkrankungen des Gastrointestinaltrakts: Gelegentlich: Mundtrockenheit. Selten: Diarrhö, Dyspepsie. Erkrankungen der Haut und des Unterhautzellgewebes: Gelegentlich: Hautausschlag. Selten: Juckreiz Skelettmuskulatur-, Bindegewebs- und Knochenerkrankungen: Selten: Muskelspasmen. Allgemeine Erkrankungen und Beschwerden am Verabreichungsort: Selten: Peripher Ödeme, Asthenie. Unmittelbar nach der Anwendung inhalativer Therapien kann paradoxer Bronchospasmus mit raschem Anstieg von Keuchen und Kurzatmigkeit auftreten. Paradoxe Bronchospasmus spricht auf Behandlung mit rasch wirksamen Bronchodilaren an und sollte umgehend behandelt werden flutiform sollte sofort abgesetzt werden. Nach einer Begutachtung des Patienten sollte gegebenenfalls mit einer alternativen Therapie begonnen werden. Oa flutiform sowohl Fluticasonpropionat als auch Formoterolfumarat enthält, kann das bei den einzelnen Wirkstoffen zu beobachtende Nebenwirkungsmuster auffreten. Die folgenden Nebenwirkungen können unter Fluticasonpropionat bzw. Formoterolfumarat auftreten, wurden aber während der klinischen Prüfung von flutiform" nicht beobachtet Fluticasonpropionat: Überempfindlichkeitsreaktionen wie Urtikaria, Pruritus, Angioödem (vorwiegend fazial und oropharyngeal), anaphylaktische Reaktionen. Insbesondere bei längerfristiger Anwendung hoher Dosen können systemische Wirkungen inhalativer Kortikosteroide auftreten. Dazu gehören Cushing-Syndrom, cushingoide Merkmale, Nebennierensuppression, Wachstumsverzögerung bei Kindern und Jugendlichen, Abnahme der Knochenmineraldichte, Katarakt und Glaukom, Blutergüsse, Hautatrophie und Infektionsneigung. Die Anpassungsähigkeit an erhöhte Belastung kann beeinträchtigt sein. Allerdings sind die oben beschriebenen Nebenwirkungen unter inhalativen Kortikosteroiden sehr viel weniger wahrscheinlic als unter oralen Kortikosteroiden. Anhaltende Therapie mit hohen Dosen inhalativer Kortikosteroide kann zu klinisch relevanter Nebennierensuppression und akuter Nebennierenkrise führen. In Situationen von besonderem Stress (Trauma, chirurgische Eingriffe, Infektion) kann eine zusätzliche Einnahme systemischer Kortikosteroide notwendig

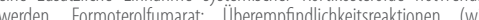
Hypoto-nie, Urtikaria, angioneurotisches Ödem, Pruritus, Exanthem) Verlängerung des QTC-Intervalls, Hypokalïmie, Übelkeit, Myalgie, Anstieg der Laktatkonzentration im Blut. Die Behandlung mit Beta2-Agonisten wie Formoterol kann zu einem Anstieg der Blutspiege von Insulin, freien Fettsäuren, Glyzerin und Ketonkörpern führen Bei Patienten, die inhalatives Natriumcromoglicat als Wirkstof anwendeten, wurde über Überempfindlichkeitsreaktionen berichtet Auch wenn flutiform nur eine geringe Konzentration an Natriumcromoglicat als Hilfsstoff enthält, ist nicht bekannt, ob Überempfindcromoglicat als Hilfsstoff enthält, ist nicht bekannt, ob Uberempfindlichkeitsreaktionen dosisabhängig sind. Im unwahrscheinlichen Fall eine sollte eine Behandlung gemäß allgemeinem Standard erfolgen. Diese sollte eine Behandlung gemäß allgemeinem Standard erfolgen. Diese kann den Gebrauch von Anthistaminika sowie andere Behandlungen beinhalten. Es kann erforderlich sein, flutiform unverzüglich abzusetzen und eine alternative Asthmatherapie einzuleiten. Dysphonie und Kandidose konnen durch Gurgein oder Mundspulungen mit Wasser Oder auch durch Zahnepuzen nach der Gabe des Produktes Behandlung mit flutiform kann die symptomatische Behandlung von Kandidosen mit lokalen Antingkotha effolgen. Warnhinweise: Fur Kinder unzugänglich aufbewahren. flutiform $50 \mu \mathrm{g} / 5 \mu \mathrm{g}$ und $125 \mu \mathrm{g} / 5 \mu \mathrm{g}$ sollen bei Kindern unter 12 Jahren NICHT angewendet werden. flutiform $250 \mu \mathrm{g} / 10 \mu \mathrm{g}$ werden. Mundipharma GmbH, 65549 Limburg. 06-15

Weitere Informationen unter: www.flutiform.de 